\title{
Study of Functional Vulnerability Status of Tehran Hospitals in Dealing With Natural Disasters
}

Batol Ahmadi ${ }^{1}$, Abbas Rahimi Foroushani ${ }^{2}$, Narjes Tanha ${ }^{1}$, Amjad Mohamadi Bolban Abad ${ }^{1}$, Heshmatollah Asadi $^{1}$

${ }^{1}$ Department of Health Management and Economics, School of Public Health, Tehran University of Medical Sciences, Tehran, Iran

${ }^{2}$ Department of Epidemiology and Biostatistics, School of Public Health, Tehran University of Medical Sciences, Tehran, Iran

\section{Type of article: Original}

\begin{abstract}
Introduction: At the time of disasters, hospitals are considered as one of the most important facilities which should provide emergency services continuously. The purpose of this study was to determine the functional vulnerability of Tehran hospitals in dealing with natural disasters.

Methods: This cross-sectional study was conducted in educational hospitals of Tehran University of Medical Science (TUMS), in 2013. Of all 26 hospitals, 12 hospitals were selected through single-stage cluster sampling, including six general (Shariati, Amiralam, Sina, Imam, Hazrat Rasoul, Baharlou) and six specialized (Bahrami, Hazrat-e Aliasghar, Shahid rajaie, Roozbeh, Moheb yas, Children Medical Center) hospitals. Data were collected using World Health Organization (WHO) checklist for functional indicators of safe hospitals, recommended for countries with similar climates. Data were collected through interviews with members of hospital crisis committees and direct observations. Data analysis was carried out using SPSS version 18 using descriptive statistics and Fisher's exact test.

Results: Fifty percent of hospitals in the study, in terms of functional vulnerability status, were in satisfactory condition with low level of vulnerability, $41.7 \%$ were in the moderate level and $8.3 \%$ were in a non-satisfactory condition with high levels of vulnerability. The results of Fisher's exact test showed that there wasn't a significant correlation between functional vulnerability and hospitals lifespan $(\mathrm{p}=0.99)$ and type of specialty $(\mathrm{p}=0.99)$.

Conclusion: As this study, assesses hospitals' main weaknesses in terms of procedures, strategies, plans, human resources, monitoring and evaluation, it is essential that each of these areas be reviewed by hospital managers separately, in order to take significant actions to eliminate their weaknesses.
\end{abstract}

Keywords: Disasters, Functional Vulnerability, Hospitals

\section{Introduction}

Disasters can happen anytime, anywhere and for anyone. At any time, individuals and communities may be affected by disasters all around the world (1). Natural disasters such as floods, earthquakes and storms often have devastating impacts on human societies and impose loss in disaster prone areas. Disasters also impose widespread economic and social problems to the civil and social infrastructure of disaster-stricken countries (2). Every year, approximately 200 million people are involved with disasters worldwide. During the past two decades, about 800 million people were affected by a disaster and three million people have lost their lives due to natural disasters (3). Also, disasterprone countries have lost about $3 \%$ of their gross domestic product (GDP) due to the loss caused by natural disasters (4). According to global statistics, Iran is one of ten disaster-prone countries in the world (5). Also, Tehran is one of the cities placed on the earthquake zone belt, and has been estimated to be stricken by earthquakes for the past three decades (6). According to predictions, if an earthquake occurs in Tehran, from the Ray fault line, almost 500,000 houses will be destroyed and nearly 400,000 people may die (6). Considering the very high population density in

\section{Corresponding author:}

Heshmatollah Asadi, Department of Health Management and Economics, School of Public Health, Tehran University of Medical Sciences. Tel: +98.9373754220, E-mail: heshmat.asadi64@gmail.com

Received: March 08, 2016, Accepted: June 15, 2016, Published: November 2016

iThenticate screening: June 15, 2016, English editing: September 15, 2016, Quality control: October 08, 2016

(C) 2016 The Authors. This is an open access article under the terms of the Creative Commons Attribution-NonCommercialNoDerivs License, which permits use and distribution in any medium, provided the original work is properly cited, the use is non-commercial and no modifications or adaptations are made. 
Tehran, the issue of disaster preparedness becomes very important. In its 2002 report, the World Health Organization called on its member states not to be satisfied just to provide health services after disasters, but also support a health strategy based on epidemiological concepts (7). Preparedness is one of the steps in coping with disasters in the crisis management cycle (8). Namely, it is necessary to be well-prepared before a disaster, by coordinating all relevant departments and holding training and practicing courses for staffs at specified intervals. For proper preparedness in the time of dealing with disasters, identifying and defining risks, establishing procedures, reviewing supportive programs and innovating performance, appropriate arrangements, are necessary (9). Results of a study conducted in Tehran showed that $56 \%$ of hospitals were more than $60 \%$ prepared for disasters, and $14 \%$ of the studied hospitals were less than $30 \%$ prepared (10). In another study, which was carried out in hospitals affiliated to Shahid Beheshti University of Medical Sciences, $86.7 \%$ of the hospitals were reported to be in good level of preparedness (11). Another research also indicated that hospitals and crisis committees' performance during crises, personnel training, hospital facilities and equipment conditions were satisfactory. Also, its results showed that hospitals preparedness, during the crisis, in terms of organizing staff, the capacity of intensive care units, communication and information systems, crisis management, and the birth and death registration system were not satisfactory (12). The vulnerability of health facilities is defined in three dimensions of structural, non-structural and functional. "The interaction between a health facility's susceptibility (inherent factors in a facility that allow hazards to cause an emergency) and resilience (the ability of the facility to withstand damages caused by an emergency) determines its vulnerability. Regarding this definition, functional vulnerability in hospital is interfacing between weakness and flexibility of functional parameters such as accessibility, communication and transportation. A health facility's preparedness for emergencies is based on a sound assessment of vulnerability" (13). After the disaster, health facilities have a great impact on socio-economic and psychological recovery (14). Because of the nature of health sector proceedings for treatment and rehabilitation of victims, it is an important part of preparedness planning and responding to disasters. At the time of disaster, health services should be provided without any interruption, therefore, health care facilities are supposed to provide services during a disaster without discontinuing. At the time of disasters, hospitals are considered as one of the most important facilities which should provide emergency services continuously (15), to reduce victim mortality and injuries (5). If before disaster, hospitals are prepared properly, they would be able to carry out their duties well (16). Since other aspects of hospital readiness, in terms of structural, non- structural (4), and knowledge of personnel (15) in other studies have been investigated, here we specifically investigate the status of hospitals' functional readiness, using tools introduced by the World Health Organization. Therefore, the aim of this study was to determine the functional vulnerability of Tehran hospitals in dealing with natural disasters.

\section{Material and Methods}

\subsection{Research design and setting}

This is a descriptive-analytic and cross-sectional study conducted in hospitals affiliated to Tehran University of Medical Science (TUMS), in 2013.

\subsection{Sampling}

\subsubsection{Sample size determination}

The population study consisted of 26 hospitals among which 12 hospitals were selected through single-stage cluster sampling, including six general (Imam, Amiralam, Hazrat Rasoul, Shariati, Sina, Baharlou) and six specialized (Bahrami, Hazrat-e Aliasghar, Moheb yas, Roozbeh, Shahid rajaie, Children Medical Center) hospitals.

\subsubsection{Sampling method}

As the checklist used in this study consisted of 17 scopes and 139 items, it would have taken a lot of time to collect them all completed in all 26 hospitals affiliated to Tehran University of Medical Sciences, therefore 12 hospitals were estimated as our sample using a proportional estimation formula. Hospitals, selected through single-stage cluster sampling and their location in Tehran, were as follows: Shariati (Center), Amiralam (Center), Sina (Center), Imam (Center), Hazrat Rasoul (Center), Baharlou (South), Bahrami (East), Hazrat-e Aliasghar (North), Shahid Rajaie (North), Roozbeh (South), Moheb Yas (Center), and Children Medical Center (Center). It may be worth saying that hospitals affiliated to Tehran University of Medical Sciences are mostly located in the center of the city.

\subsection{Instrument}

Data were collected by functional indicators of a safe hospitals checklist, recommended by the WHO for countries with similar climates (17). The checklist consisted of 17 scopes including: location (three items), accessibility (12 items), internal circulation (four items), interoperability (six items), equipment and supplies (10 items), standard operating procedures (SOP) protocols (four items), strategies (nine items), guidelines (seven items), logistic system 
(eight items), public utility resource (15 items), safety and alarm systems (11 items), security systems (five items), communications and transportation systems (four item), public information systems (nine item), plans for emergency and disaster (18 items), human resources (11 items), monitoring and evaluation (three items) in a total of 139 questions. In each scope and in general, functional vulnerability condition is evaluated in three levels: low vulnerability (satisfactory), moderate vulnerability, and high vulnerability (unsatisfactory). Overall, attaining at least two-thirds of the total scores (at least 185.3) indicates low vulnerability (satisfactory), one-half to two-thirds of the total scores (143.5-185.3) indicates moderate vulnerability, and less than one-half of the total scores (less than 143.5) indicates high vulnerability (unsatisfactory).

\subsection{Data collection}

First we were given permission to collect data in hospitals from hospital chief managers, then we began completing checklists through interviewing 48 members of different crisis committees. In the final step we also collected data through 12 direct observations of hospitals and compared and adjusted them with the information of completed checklists.

\subsection{Statistical analysis}

The data were analyzed using SPSS software version 18 by descriptive statistics and Fisher's exact test.

\section{Results}

Among the studied hospitals, functional vulnerabilities status of one hospital (A), with the lowest average score of 139, was unsatisfactory, five hospitals (B, C, D, F, L) were assessed moderate, and six hospitals (E, G, H, I, J, K) were in satisfactory condition in terms of functional vulnerability (In the present study, at the request of hospital chief managers, we did not name the hospitals and had to use letters instead). One hospital (E), with the highest average score of 215, was the best with the lowest level of functional vulnerability. On the whole, functional vulnerability status for 12 studied hospitals, with average of 181.9, was indicated as moderate. In total, in terms of functional vulnerability status, 50 percent of hospitals in the study (six hospitals), were in satisfactory condition with a low level of vulnerability, $41.7 \%$ (5 hospitals) were in the moderate, level and one hospital (8.3\%) was in a non-satisfactory condition with high level of vulnerability (Table 1). In the scope of location, all hospitals were in satisfactory condition, but in the scope of planning for emergency and disaster, only one hospital was assessed in moderate condition, the rest were in unsatisfactory condition (Table 2). The results of Fisher's exact test showed that there wasn't a significant correlation of functional vulnerability with hospitals lifespan $(\mathrm{p}=0.99)$ and type of specialty $(\mathrm{p}=0.99)($ Table 3$)$.

Table 1. Functional vulnerability condition of 12 responding hospitals

\begin{tabular}{|l|l|l|}
\hline Hospital & Sum of Scores & Vulnerability condition \\
\hline A & 139 & High (unsatisfactory) \\
\hline B & 170 & Average (moderate) \\
\hline C & 182 & Average (moderate) \\
\hline D & 149 & Average (moderate) \\
\hline E & 215 & Low (satisfactory) \\
\hline F & 151 & Average (moderate) \\
\hline G & 214 & Low (satisfactory) \\
\hline H & 211 & Low (satisfactory) \\
\hline I & 200 & Low (satisfactory) \\
\hline J & 189 & Low (satisfactory) \\
\hline K & 204 & Low (satisfactory) \\
\hline L & 158 & Average (moderate) \\
\hline Average of all hospitals & 181.9 & Average (moderate) \\
\hline
\end{tabular}


Table 2. Functional vulnerability condition of 12 responding hospitals

\begin{tabular}{|l|l|l|l|l|}
\hline \multirow{2}{*}{ Variables } & Satisfactory & Moderate & Unsatisfactory & \multirow{2}{*}{ General condition } \\
\cline { 2 - 5 } & $\mathrm{n}(\%)$ & $\mathrm{n}(\%)$ & $\mathrm{n}(\%)$ & \\
\hline Location & $12(100)$ & $0(0)$ & $0(0)$ & Good \\
\hline Accessibility & $7(58.4)$ & $1(8.3)$ & $4(33.3)$ & Good \\
\hline Internal Circulation & $12(0)$ & $0(0)$ & $0(0)$ & Good \\
\hline Interoperability & $11(91.7)$ & $0(0)$ & $1(8.3)$ & Good \\
\hline Equipment and Supplies & $12(100)$ & $0(0)$ & $0(0)$ & Good \\
\hline Standard Operating Procedures ... & $3(25)$ & $5(41.7)$ & $4(33.3)$ & Moderate \\
\hline Procedures & $1(8.3)$ & $1(8.3)$ & $10(83.4)$ & Weak \\
\hline Guidelines & $1(8.3)$ & $0(0)$ & $11(091.7$ & Weak \\
\hline Logistic System & $8(66.7)$ & $4(33.3)$ & $0(0)$ & Good \\
\hline Public utility resource & $6(50)$ & $5(41.7)$ & $1(8.3)$ & Good \\
\hline Safety and Alarm Systems & $9(75)$ & $3(25)$ & $0(0)$ & Good \\
\hline Security Systems & $12(0)$ & $0(0)$ & $0(0)$ & Good \\
\hline Communications and Transportation & $10(83.4)$ & $0(0)$ & $2(16.6)$ & Good \\
\hline Public Information Systems & $8(66.7)$ & $2(16.6)$ & $2(16.6)$ & Good \\
\hline Plans for Emergency and Disaster & $0(0)$ & $1(8.3)$ & $11(97.7)$ & Weak \\
\hline Human Resources & $2(16.6)$ & $2(16.6)$ & $8(66.7)$ & Weak \\
\hline Monitoring and Evaluation & $4(33.3)$ & $1(8.3)$ & $7(58.4)$ & Weak \\
\hline All domains (scope) & $6(50)$ & $5(41.7)$ & $1(8.3)$ & Moderate \\
\hline
\end{tabular}

Table 3. Frequency distribution of functional vulnerability of responded hospitals according to the lifespan and type of specialty

\begin{tabular}{|l|l|l|l|l|}
\hline Variable & Functional vulnerability & Satisfactory \& Moderate & Unsatisfactory & p-value \\
\hline \multirow{3}{*}{ Hospitals lifespan } & $35>$ & $3(25)$ & $0(0)$ & $0.99^{* *}$ \\
\cline { 2 - 4 } & $35<$ & $8(66.7)$ & $1(8.3)$ & \\
\cline { 2 - 4 } & Total & $11(91.7)$ & $1(8.3)$ & \\
\hline \multirow{3}{*}{ Type of hospitals } & Specialized & $6(50)$ & $0(0)$ & \multirow{2}{*}{$0.99^{* *}$} \\
\cline { 2 - 4 } & General & $5(41.7)$ & $1(8.3)$ & \\
\cline { 2 - 4 } & Total & $11(91.7)$ & $1(8.3)$ & \\
\hline
\end{tabular}

*We merged Moderate \& Satisfactory in one group

** Results based on Fisher's exact test

\section{Discussion}

The findings of our study showed that functional vulnerability of Tehran hospitals was moderate. In other studies conducted in Iranian hospitals, functional preparedness of hospitals in Kermanshah (18) Tehran (19), Northern provinces of Iran (20) and overall Iranian hospitals (2) were reported to be in an average level. Although Iran is one of the ten countries in the disaster-prone zone (5) and Tehran, as the largest populated city, stands as one of the most disaster-prone cities, (6) the level of preparedness is not satisfactory. In all of these studies, several suggestions have been proposed on preparing hospitals to cope with disaster. The important issue is that in all of these studies, the will and intention was to improve the situation and increase the level of hospitals' preparedness in the time of facing disasters. Also, the results of our study showed that the studied hospitals were in good condition, in scopes of location, accessibility, internal circulation, interoperability, equipment and supplies, logistic system, public utility resource, safety and alarm systems, security systems, communications, transportation systems, and public information, which means that these hospitals are in a good level of infrastructure and hardware. A research done in Japan showed that hospitals were promising in terms of handling and transportation, for dealing with crisis situations. Also, approximately all hospitals had notable stores of amenities and facilities such as tents, water reservoirs, folding beds, generators, etc. (14). In the study of Salari et al., Shiraz hospitals had good emergency response plans in terms of admissions, training and maintenance, but were in a moderate level in terms of safety, communications, and transportation, and evacuation conditions (21). In the study of Amiri et al., the mean percentage score of hospitals in management of the emergency disasters plans in the Northern Iran was reported satisfactory, but the mean score of hospitals' plans to support vital services, instrument, and hazardous substances safety in disasters was moderate (20). A Study of Hekmatkhah et al. showed that hospitals' preparedness level in Orumieh University of Medical Sciences was in moderate level in dimensions of vital services support, and in low 
level of preparedness in terms of safety of equipment and hazardous materials, planning of needed equipment and consuming goods (22).

The results of our study showed that studied hospitals were in a weak condition in five domains including procedures, guidelines, plans for emergency and disaster, human resources, monitoring and evaluation. Hekmatkhah et al. findings indicated that some weaknesses such as lack of planning for using emergency electric generators can be seen in some hospitals during the crisis. Also, in his study, planning for hospital evacuation, setting up outdoor field hospital and training were reported to be very poor (22). Results of a Nasiri-pour et al. study revealed that border hospitals in Kermanshah Province had a medium level of preparedness in dimensions of organizing hospital personnel, and low level of preparedness in maneuvering and practicing to deal with crisis (18). But in a Salari et al. study, Hospitals in Shiraz were in a good condition of preparedness in dimensions of human workforce, leadership and management (21). In an Amiri et al. study, scopes including hospital educational program, environmental health measures, reducing dangers related to hospital buildings, and evacuation actions were ranked weak (20). The results of our study are not consistent with the study conducted in Japan in which most hospitals had education or training programs for emergency medical services and had conducted disaster maneuvers for hospital staff at least once a year. One hospital in Tokyo conducts disaster maneuvers not only involving hospital staff but also patients as well. Also, most of the Japanese hospitals had been found to be prepared for disasters in terms of enhancing the capacity of their human resources. However, in the study conducted in Japan out of 14 hospitals, 13 hospitals had structural deficiencies of buildings for being prepared for disasters. Results of the Japanese study also indicated that while $80 \%$ of studied hospitals had special spaces available for evacuating patients during emergencies, the rest of these hospitals $(20 \%)$ did not have such spaces, so they were not able to accommodate large numbers of evacuees during emergencies. (14). Hospitals in our study were ranked as weak preparedness hospitals for managing emergency and disaster in domains of procedures, guidelines, plans, human resources, monitoring and evaluation. Given that in formulating effective disaster recovery plans for hospitals identifying and defining the risks, defining procedures, reviewing supportive plans, having innovative performance, and planned arrangements are required (9), it is recommended that hospitals formulate specific plans for all stages of managing disasters including before, during and after the disaster. Hospitals should also promote their internal planning in the situation of dealing with disaster. In the studied hospitals, human resources were also in poor condition. It should be noted, as emphasized in various studies, well trained personnel is the most important factor in managing and response to disasters and crises. Unavailability or lack of well-trained personnel disrupts the process of providing care for the injured, and hiring personnel from elsewhere, to sustain providing appropriate care services, would add additional economic burden (23). Therefore, it is recommended that hospitals formulate procedures for designing labor force and responsibilities, as well as formulating procedures for using and organizing volunteers in critical conditions. Furthermore, it is recommended that main and alternative teams should be appointed, and their members should be sent, under the superintendence of the Red Crescent, to internal and external crises in order to gain experience. Hospitals' authorities not only should formulate their human resource charts in facing disasters, but also should held regular and periodic maneuvers, according to this chart, to be prepared for possible disasters. Afterwards, managers should review maneuver performances and give feedback to their personnel.

According to our study results, contrary to what was expected, there was not any significant correlation between functional vulnerability and hospital lifespan. According to the Zangabadi and Esmailian (24) study, one of the most important factors affecting buildings' vulnerability in facing disasters is buildings' lifespan. This discrepancy can be due to the few numbers of studied hospitals in our study. Also no significant correlation was observed between hospital functional vulnerability and type of hospital specialty. This finding is consistent with results of Hekmatkhah $\&$ et al. study (22). In the present study, at the request of hospital chief managers, we did not name the hospitals and had to use letters instead. The main limitation of the study, which led to waste of money and time, was the difficulty in collecting the required data, which in some cases, researchers faced problems collecting completed checklists due to lack of proper cooperation of hospital related authorities.

\section{Conclusions}

Results of the present study showed that operational readiness status of studied hospital for natural disaster is in the medium level. However hospitals were shown to be weak in some scopes such as procedures, strategies, plans, human resources and monitoring and evaluation. It is recommended that hospital authorities define a precise and specific plan for the entire process of facing disasters including stages before, during and after the disaster, develop their internal planning and formulate a procedure for designing responsibilities of personnel and volunteer 
individuals in critical situations. Although results showed that hospitals' lifespan and specialty had no impact on their readiness to deal with critical situations, it is recommended that more studies should be carried out in this field.

\section{Acknowledgments:}

This article has been derived from a thesis entitled "Study of functional vulnerability status of Tehran hospitals in dealing with natural disasters" in Master of Science Degree which is executed with the support of Tehran University of Medical Sciences.

\section{Conflict of Interest:}

There is no conflict of interest to be declared.

\section{Authors' contributions:}

All authors contributed to this project and article equally. All authors read and approved the final manuscript.

\section{References:}

1) Marquez MR. A Perspective on Global Disaster Behavioral Health Response. International journal of Science Commerce and Humanities. 2014; 2(3): 257-65.

2) Daneshmandi M, Amiri H, Vahedi M, Farshi M, Saghafi A, Zigheymat F. Assessing the level of preparedness for confronting crisis such as flood, earthquake, fire and storm in some selected hospitals of Iran. Military Medicine Journal. 2010; 12(3): 167-71.

3) Mastaneh Z, Mouseli L. Capabilities and limitations of crisis management in the teaching hospitals of Hormozgan University of Medical Sciences, 2010. Sci Res Essays. 2013; 8(26): 1196-202. doi: 10.5897/SRE11.1668.

4) Malekshahi F, Mardani M. Abilities and limitations of crisis management in Shohadaye Ashayer and Social Security hospitals of Khorramabad in 2007. Journal of Critical Care Nursing. 2009; 1(1): 29-34.

5) Arab M, Zeraati H, Akbari Haghighi F, Ravangard R. A Study on the executive managers' knowledge and performance, and their hospitals preparedness against earthquake events and their relationships at public hospitals (affiliated by tehran university of medical sciences); 2005-2006. Journal of Health Administration. 2009; 11(34): 7-14.

6) Karimi HR, Karimi AR. Identification of Factors affecting human resource management in Imam Sajjad Hospital. In: 3, editor. Conference on Preventive Medicine and Public Health. Bandarabas. 2010; 230-7.

7) WHO. Rapid health assessment protocols for emergencies. Translated to Persian by: Mamishi S, Sharifi I, Keshavarz H. Tehran: Ministry of Health \& Medical Education Health Subcommittee for Natural Disasters Reduction \& WHO press. 2001.

8) Shreve C, Fordham M, Anson S, Watson H, Hagen K, Wadhwa K, et al. Report on risk perception and preparedness. 2014.

9) Çalin E, Kaya H, Çavuşoğlu A, Annuş S, Şen B. Disaster Readiness of Hospital Information Systems: A Case Study from a Turkish University Hospital. 6th International Information Security \& Cryptology Conference; Ankara. 2013; 236-41.

10) Maher A, Yeganeh SM, Lari MA, Sayedin SH. The study of the quality and capacity of equipments' functionality and non-structural vulnerability in selected Tehran general hospitals during an earthquake. Int J Health Syst Disaster Manage. 2014; 2(2): 73-131. doi: 10.4103/2347-9019.139041.

11) Hosseini Shokouh SM, Anjomshoa M, Mousavi SM, Sadeghifar J, Armoun B, Rezapour A, et al. Prerequisites of preparedness against earthquake in hospital system: A survey from Iran. Glob J of heal sci. 2014; 6(2): 237-45. doi: 10.5539/gjhs.v6n2p237. PMID: 24576386, PMCID: PMC4825456.

12) Zaboli R, Sajadi HS. Assessing hospital disaster preparedness in Tehran: Lessons learned on disaster and mass casualty management system. Int J Health Syst Disaster Manage. 2014; 2(4): 193-248. doi: 10.4103/2347-9019.144405.

13) Organization WHO. Field manual for capacity assessment of health facilities in responding to emergencies. Manila: World Health Organization, 2006. Available from: http://apps. who.int/bookorders/anglais/detart1.jsp?sesslan=1\&codlan=1\&codcol=52\&codcch=98\#

14) Mulyasari F, Inoue $S$, Prashar S, Isayama $K$, Basu $M$, Srivastava $N$, et al. Disaster preparedness: Looking through the lens of hospitals in Japan. International Journal of Disaster Risk Science. 2013; 4(2): 89-100. doi: 10.1007/s13753-013-0010-1. 
15) Lakbala P. Hospital Workers Disaster Management and Hospital Nonstructural: A Study in Bandar Abbas, Iran. Glob J Health Sci. 2015; 8(4): 221-6. doi: 10.5539/gjhs.v8n4p221. PMID: 26573039, PMCID: PMC4873581.

16) Sobhani G, Khammarnia M, Hayati R, Ravangard R, Heydari AR, Heydarvand S. Investigation of the preparedness level of the hospitals against disasters in Bandar Abbas, Iran, in 2012. J Pak Med Assoc. 2014; 64(5): 506-9. PMID: 25272533.

17) WHO. Safe hospitals in emergencies and disasters: structural, non-structural and functional indicators. Geneva. World Health Organization. 2010.

18) Nasiripour A, Raeissi P, Mahbobi M. Border hospital readiness in handling border related crisis in Kermanshah Province , Iran, 2007. Journal of Health Administration. 2007; 10 (28): 41-8.

19) Zaboli RA, Tofighi Sh, Amerion A, Moghaddasi H. Survey of Tehran City Hospitals Preparedness for Disaster. J Mil Med. 2006; 8(2): 103-11.

20) Amiri M, Chaman R, Raei M, Shirvani SD, Afkar A. Preparedness of hospitals in north of iran to deal with disasters. Iran Red Crescent Med J. 2013; 15(6): 519-21. doi: 10.5812/ircmj.4279. PMID: 24349752, PMCID: PMC3840841.

21) Salari H, Esfandiari A, Heidari A, Julaee H, Rahimi SH. Survey of natural disasters preparedness in public and private hospitals of Islamic republic of Iran (case study of shiraz, 2011). Int J Health Syst Disaster Manage. 2013; 1(1): 1-58. doi: 10.4103/2347-9019.122441.

22) Hekmatkhah A, Rahimi H, Kamali Aghdam M, Taghavi shahri SM, Sadeghifar J, Hamozadeh P. Assessing The Preparedness Rate Againest Earthquake Risk In Hospitals Affiliated To Urmia University Of Medical Sciences; 2011. Journal of Urmia Nursing and Midwifery Faculty. 2012; 10(2): 0-0.

23) Banatin CA, Go MV, Peňafiel AMRM, Bituin RA. Safe Hospitals in Emergencies and Disasters: Philippine Indicators. Administrative Order. 2009.

24) Zangiabadi A, Esmaeilian Z. The analysis of indicators of vulnerability of urban housing against the risk of natural disasters (Case Study: housing in Isfahan). Geography and Environmental Hazards. 2013; 1(4): 113-29. 